\title{
绿色环境暴露对居民心理健康的影响研究 以南京为例
}

\author{
李智轩,何仲禹, 张一鸣, 金霜霜, 王雪梅, 朱捷, 刘师岑 \\ (南京大学建筑与城市规划学院, 南京 210093)
}

\begin{abstract}
摘要: 绿色环境暴露对心理健康的影响长期以来都受到国内外不同领域学者的关注,但从个体与环境交互的角度 关注衡量个体对环境实际感知的研究较少。论文试图基于对居民视觉感知和时空活动等因素的考虑, 结合机器学 习等技术扩展绿色环境暴露的测度方式, 并构建绿色环境暴露对心理健康影响的概念框架。同时, 以南京为实证 对象, 运用结构方程模型对比分析绿地率、绿视率、绿色视觉暴露对心理健康影响的差异。研究发现, 3 种绿色环 境暴露测度指标对心理健康均有显著正向影响, 但影响程度和路径存在差异, 建立更加综合的绿色环境暴露评价 指标体系至关重要。主观建成环境可以作为绿视率和绿色视觉暴露对心理健康影响的中介变量, 身体活动仅作为 绿色视觉暴露影响心理健康的中介变量。研究拓展了绿色环境暴露对心理健康影响的研究框架, 并对城市绿地系 统的规划管理具有参考价值。
\end{abstract}

关键词: 环境暴露; 绿色视觉暴露; 绿视率; 心理健康; 机器学习;南京

对环境暴露的研究最初源于医学对职业病和 流行病的研究 ${ }^{[1]}$ 。近年来, 随着人们对环境问题的 日益重视,环境暴露的概念在交通、城市地理学、城 市规划等诸多领域都得到了发展和应用 ${ }^{[2-3]}$ 。个体 层面,包括肥胖、呼吸系统疾病和各类精神疾病在 内的居民健康问题唤起了社会对环境暴露与居民 健康联系的关注 ${ }^{[4-6]} 。 2018$ 年 Marco Helbich 在欧洲 主持开展了 NEEDS(Dynamic Urban Environmental Exposures on Depression and Suicide)项目, 旨在研 究环境暴露对抑有症等心理健康问题的影响。绿 色和蓝色环境 ${ }^{[7]}$ 、气象因素 ${ }^{[8]}$ 、噪音 ${ }^{[9]}$ 、空气污染 ${ }^{[10]}$ 等 环境因素的暴露已经被证实对居民的心理健康有 直接影响。交通出行层面, 尽管更高的体力活动水 平往往对健康有促进作用, Huang 等 ${ }^{[1]}$ 指出步行与 自行车出行可能带来更高的空气污染暴露, 对健康 产生负面影响。陈曦等 ${ }^{[12]}$ 从城市步行环境和污染 强度空间关系的角度进行了进一步的讨论。还有
部分学者研究了极端温度环境暴露对健康的影响, 发现长时间的高温或者严寒环境暴露与居民多种 疾病的发病率以及死亡率密切相关 ${ }^{[13-15]}$ 。上述研究 主要从呼吸、皮肤接触和听觉等方面分析环境暴露 对健康的影响。

另一方面, 绿色环境和公共健康的关联一直是 城市规划学界关注的重点问题。有研究表明, 暴露 在绿色环境中会对心理健康产生积极影响 ${ }^{[16-17]}$ 。这 些积极影响包括提高幸福感 ${ }^{[18]}$ 、减少焦虑 ${ }^{[19]}$ 、缓解压 力 ${ }^{[20]}$ 、提高睡眠质量 ${ }^{[2]}$ 等。传统对绿色环境的测度 主要包括数量、面积、可达性以及空间形态等方 面 ${ }^{[22-23]}$ 。然而仅对绿色环境的二维指标进行分析忽 略了个体与环境的互动关系, 难以满足更加精细化 的城市研究需求 ${ }^{[24]}$ 。国外对三维绿色环境与心理 健康的关注较早, 尤其是日本相关研究和规划实践 都较为完善 ${ }^{[25]}$ 。1 1987 年日本学者青木阳二 ${ }^{[26]}$ 首次提 出了“绿视率” 的概念, 定义绿视率为人眼观察到的

收稿日期 : 2019-04-11; 修订日期: 2019-07-18。

基金项目: 国家自然科学基金项目(51678288)。[Foundation: National Natural Science Foundation of China, No. 51678288. ]

第一作者简介:李智轩(1996-), 男, 山西太原人, 硕士生, 主要研究方向为居民时空间行为、智慧城市等。

E-mail: 771445516@qq.com

*通信作者简介: 何仲禹(1983-), 男, 辽宁本溪人, 副教授, 主要研究方向为城市住房、健康城市等。E-mail: hezy@nju.edu.cn 
图像中绿色所占的面积百分比。随后, 大阪、京都、 池田等地区政府相继推出了绿视率相关的计划和 调研报告。2004年, 日本通过了 “景观绿三法”, 正 式将绿视率作为城市景观控制的主要指标之一。 日本国土交通省公开的调查显示, 高绿视率可以带 来湿润、舒适等一系列积极的心理感受 ${ }^{[2]}$ 。处于高 绿视率环境的受访者会感觉到更加平静和安静, 感 觉时间过得更快 ${ }^{[28]}$ 。有研究进一步提出了“全方位 绿视率” 的概念, 用来对植物种类进行评估, 扩展了 绿视率的测度范围, 但实际上一定程度上将研究主 要关注点由个体感知重新变为了对客观空间的测 度 ${ }^{[29]}$ 。国内相关研究则起步较晚, 主要关注于绿视 率特征和影响因素的分析 ${ }^{[30-32]}$, 与居民健康的关联研 究比较缺乏。研究表明, 高绿视率的街道可以代替 公园绿地等场所,成为城市居民释放压力的空间 ${ }^{[33]}$ 。

尽管对绿视率的研究关注了人对绿色环境的 视觉感受, 但其本质上仍是对客观环境的直接测 度, 没有将个体实际接收到的绿色环境暴露纳人考 量。随着对个体关注的加强, 很多学者开始意识到 仅从静态空间的角度研究环境暴露存在很大不足, 个体活动的差异往往决定了环境对个体的影响程 度差异 ${ }^{[34]}$ 。早期有学者研究揭示了暴露时间与心 理健康的相关性: 访问绿地频率等因素与个体压力 感知相关 ${ }^{[35-36]}$; 住宅周边绿地的停留时间对心理健 康有显著影响 ${ }^{[37]}$ 。上述研究关注了固定活动地点 的绿色暴露, 对日常活动路线中的环境暴露考虑较 少。Zhang 等 ${ }^{[38]}$ 将绿色空间的概念进行了拓展, 从 静态空间与个体活动结合的角度提出了“绿色空间
暴露” 的概念, 并从身体健康、心理健康、社会健康 3 个方面分析了绿色空间暴露对个体健康的影响。

综上所述,现有研究主要从个体与环境中的一 个方面分析绿色环境暴露对心理健康影响,如基于 个体的停留时间、访问频率，以及基于环境的绿地 占比、绿地可达性等,较少基于环境与个体交互角 度的探索。目前, 关于绿色环境暴露的测度还没有 一个相对统一的标准, Gascon 等 ${ }^{[39]}$ 在对 28 篇相关文 献进行综述分析后, 发现绿色空间与心理健康的关 联有限, 并指出绿色空间暴露测度的差异可能是导 致评价结果不同的重要原因。因此, 本文试图从以 下方面进行研究: (1) 从个体与环境互动的角度, 建 立关于绿色环境暴露对健康的研究方法框架; (2) 结 合问卷调查数据、腾讯街景数据和遥感图像数据, 利用结构方程模型分析不同绿色环境暴露测度对 居民心理健康的影响效应和影响机制。

\section{1 绿色环境暴露的测度和对心理健康 的影响}

\section{1 绿色环境暴露影响心理健康的概念框架}

基于上述分析, 本文从个体和环境交互的角度 扩展绿色环境暴露影响心理健康的研究概念框架, 将绿色环境的暴露测度分为了单一环境视角、单一 个体视角以及个体与环境交互的视角(图 1)。其中: 单一环境视角主要基于绿地的空间属性及特征,包 括对绿地系统整体的测度, 如绿地率、绿地破碎度, 以及对绿色环境本身的测度, 如绿视率、植被类型

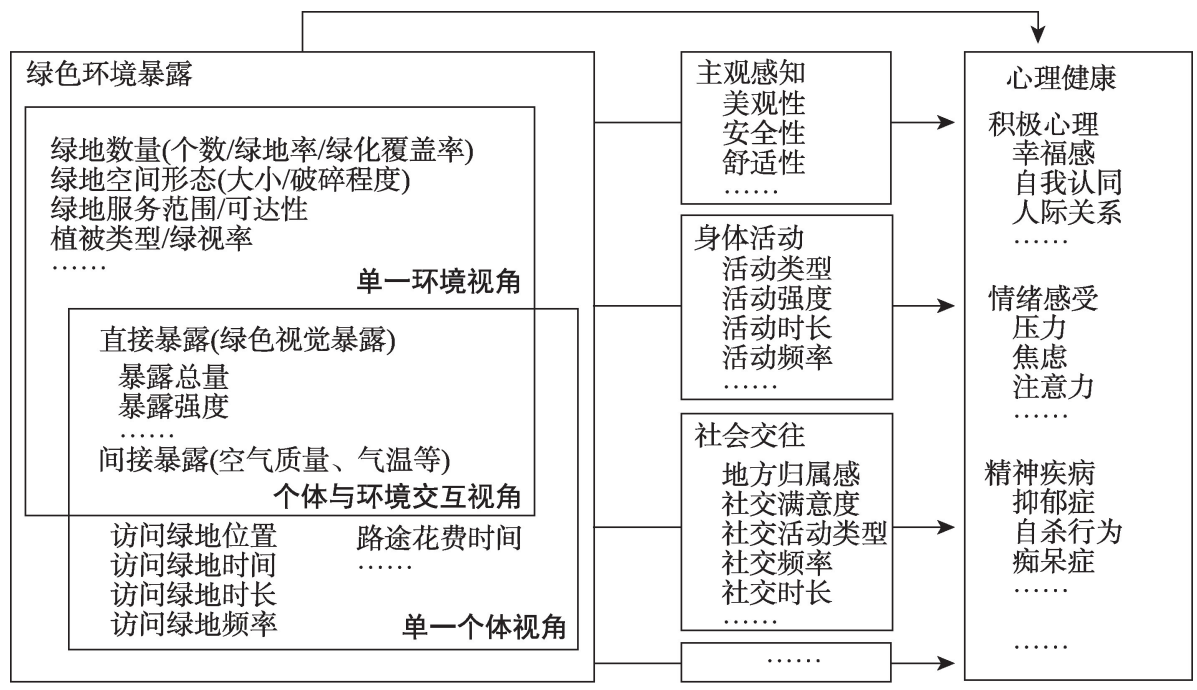

图 1 绿色环境暴露影响心理健康的概念框架

Fig.1 Aconceptual framework of greenspace exposure affecting mental health 
等。环境视角下不仅仅包含对传统意义上的绿地, 也包含对街道、建筑周边等多种环境中绿色环境的 测度。单一个体视角主要关注居民访问绿地的时 空行为,包括访问绿地本身的时空行为以及路途中 的时空行为。个体与环境交互的视角结合了客观 环境和个体时空行为, 分析个体在特定的时空环境 下接收到的环境信息, 即环境暴露。绿色视觉暴露 属于直接暴露, 可以分为暴露总量和暴露强度。暴 露强度指居民在绿色环境中瞬时的暴露水平, 绿色 环境本身、个体接触环境的时间、个体接触环境时 的状态都可能影响暴露强度; 暴露总量是一段时间 内暴露强度的累积。

心理健康可以从不同角度使用相应的量表进 行测度 ${ }^{[40]}$ 。精神疾病是反映心理健康最直接的表 现, 较为常见的有对抑郁症群体、老年痴呆症群体、 自杀人群的关注 ${ }^{[41-43]}$ 。对精神疾病的观察可以通过 观察药物使用情况间接得到 ${ }^{[4]}$ 。情绪感受是个体 短时间心理状况的直接反映, 主要分为对个体负面 情绪的关注, 即分析绿色环境对焦虑、压力等负面 情绪消除的影响 ${ }^{[45-46]}$, 以及对正面情绪的关注, 如宁 静、放松的心情 ${ }^{[47]}$ 。这类研究主要关注较短时间内 情绪的变化, 或者对一段时间内某种情绪出现频率 的回忆调查。积极心理(mental wellbeing)主要指心 理健康的积极方面, 如自我认同、幸福感等。世界 卫生组织对健康的定义中包含了对积极心理的关 注, 而不是仅仅考虑精神疾病。

绿色环境暴露除了可以直接影响心理健康外, 还可能通过多种路径间接影响心理健康。身体活 动、社会交往、与自然互动等因素都可能作为绿色 环境暴露对心理健康影响的中介因子[48-49]。已有研 究利用不同类型和尺度的指标分析了绿色环境暴 露影响心理健康的一条或多条路径, 但是缺乏对不 同指标影响路径差异的探讨, 尤其是不同视角指标 间的差异 ${ }^{[50-52]}$ 。值得说明的是, 环境对个体的影响 是一个复杂的交互作用过程, 并非是简单的单向作 用，因此中介因子之间也可能存在相互影响关系， 例如主观感知可能影响个体的身体活动和社会交 往。个人和家庭属性以及容积率、道路密度等其他 环境因素可以作为控制变量纳人框架。

\section{2 绿色视觉暴露的测算}

本文提出一种个体与环境交互视角下绿色视 觉暴露的测算方法。近年来, 新的数据获取和分析 方法的应用为准确测度绿色环境暴露提供了更多 可能 ${ }^{[33-54]}$ 。除了传统定点拍照的测量方法外, 有学
者利用机载激光雷达快速地获取大范围的 $3 \mathrm{D}$ 绿色 信息, 并推算地面上观察到的绿视率 ${ }^{[5]}$ 。街景图片 可以用来模拟居民视觉感知, 且具有数据获取简 便、数据量大、含有位置坐标等优点 ${ }^{[6]}$ 。Helbich 等 ${ }^{[5]}$ 利用腾讯街景数据和机器学习的方法, 对街景图像 中绿色和蓝色空间与老年人抑郁症的关系进行了 分析。本文主要基于绿视率和个体日间活动路线 测算绿色视觉暴露,具体计算方法如下:

首先, 定义每个观测点的绿视率为 4 个观察方 向的绿视率平均值。由于网络调用稳定性较差, 存 在部分观测点图片不足 4 张的情况, 缺失图片的绿 视率记为 0 。单个观测点绿视率计算公式如下:

$$
G_{v}=\frac{G_{v \mathrm{~N}}+G_{v \mathrm{E}}+G_{v \mathrm{~S}}+G_{v \mathrm{~W}}}{m}
$$

式中: $G_{v}$ 表示观测点的绿视率; $G_{v \mathrm{~N}} 、 G_{v \mathrm{E}} 、 G_{v \mathrm{~S}}$ 、 $G_{v \mathrm{~W}}$ 分别表示正北、正东、正南、正西四个方向街景 图片的绿视率; $m$ 表示有效图片数量 $(m \leqslant 4)$ 。

进一步可以计算得到单条路径上或一定范围 内的平均绿视率, 并近似代表绿色视觉暴露强度, 计算公式如下:

$$
\overline{G_{v}}=\frac{\sum_{i=1}^{N} G_{v i}}{N}
$$

式中: $\overline{G_{v}}$ 表示平均绿视率; $G_{v i}$ 表示单张图片绿视 率; $N$ 表示观测点 $i$ 数量。

当式(2)计算对象为居民个体活动路线时, 可以 得到居民活动路线上的平均绿视率。同时,绿色视 觉暴露不仅与居民活动空间的平均绿视率相关, 还 与居民在环境中停留时间相关, 因此, 本文定义居 民绿色视觉暴露的计算公式为:

$$
Q_{g}=\sum_{i=1}^{n} \bar{G}_{v_{i}} t_{i} x_{i}
$$

式中: $Q_{g}$ 表示居民单日绿色视觉暴露; $\overline{G_{v_{i}}}$ 表示居 民单次日间出行路线上的平均绿视率; $t_{i}$ 表示居民 单次日间出行时间; $n$ 表示居民每日出行次数; $x_{i}$ 为 有效系数,与居民出行时间、交通方式、速度等因素 有关, 例如夜间有效系数小于日间, 乘车时有效系 数小于步行时。限于数据的可获取性, 本文定义的 绿色视觉暴露只包含居民活动路线上的暴露, 没有 将居民停留点(如小区、工作地、公园等内部)和周边 的绿色环境暴露纳人计算。

\section{3 街景图片绿视率识别}

获取居民的绿视率是测算上述绿色视觉暴露 的关键。传统研究主要通过 Photoshop、GIMP 等软 
件手动统计图片中绿色像素所占比例来计算绿视 率, 难以实现大规模处理 ${ }^{[25,30]}$ 。也有研究利用 HSV 色彩空间结合程序代码实现了自动识别绿色像素, 但容易将非自然环境的绿色物体(如汽车、广告牌) 纳人绿视率中, 具有较大的测量误差 ${ }^{[8]}$ 。近年来, 机器学习技术使研究者可以更准确、快速、细致地 估算图片绿视率, 尤其是图像分割技术的发展极大 地推动了相关领域的研究 ${ }^{[77]}$ 。

本文主要利用Python 中的 Keras 库搭建卷积神 经网络(Convolutional Neural Networks, CNN) 来识 别图片中的绿视率。 $\mathrm{CNN}$ 是深度学习的主要算法 之一, 一般由输人层、隐含层和输出层 3 个部分组 成, 其中隐含层一般包含卷积层、池化层、激励层和 全连接层等结构。CNN模型在遥感、城市特征识别 等领域都有广泛应用 ${ }^{[59-61]}$ 。图 2 显示了该模型的具 体结构。模型主要由 4 层卷积层(Conv2D)、3 层池 化层(MaxPooling2D/AveragePooling2D)和 2 层全连 接层(Dense)组成, 并加人 Flatten 层用于将图像一维 化, 以及 5 层随机失活层(Dropout)防止模型过拟合 (表 1)。与 FCN、SegNet等利用了反卷积结构的语 义分割方法相比, 该模型将单一标签作为输出层, 具有较为简单、运算速度快、对训练集的要求更低 等优点, 且对分析居民感知等较为抽象的内容有更 好的效果。为了简便计算, 模型输人层设置为 $224 \times$ 224 像素大小的正方形图像; 输出层为分类标签, 即 图片绿视率值。

随机选取南京市 1200 张街景图像作为训练样 本。为获得更为准确的训练数据, 研究使用了 SegNet网站中提供的模型对样本中的图像进行语义分

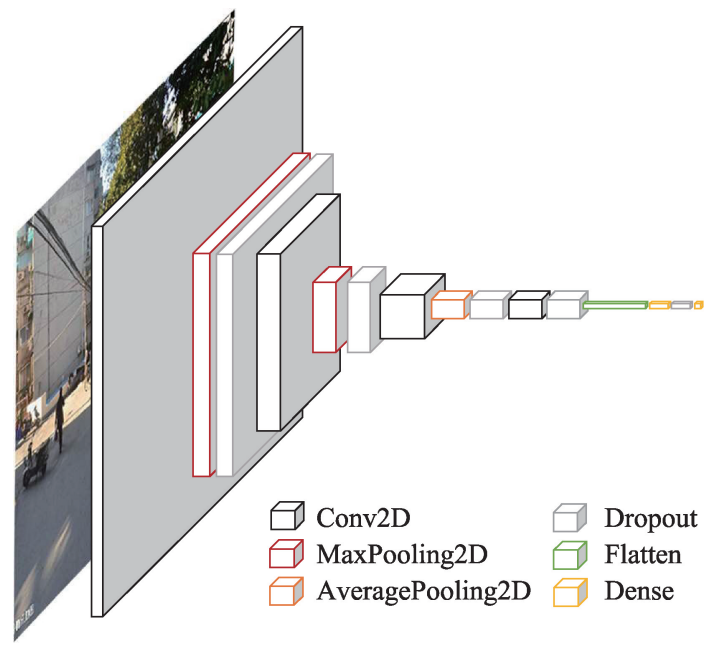

图 2 卷积神经网络模型结构

Fig.2 Convolutional neural network model structure
表 1 卷积神经网络模型参数

Tab.1 Convolutional neural network model parameters

\begin{tabular}{lcl}
\hline \multicolumn{1}{c}{ 层级结构 } & 卷积核尺寸 & 输出图片尺寸 \\
\hline Input & & $224 \times 224 \times 3$ \\
Conv2D & $3 \times 3$ & $222 \times 222 \times 32$ \\
MaxPooling2D & $2 \times 2$ & $111 \times 111 \times 32$ \\
Dropout & & $111 \times 111 \times 32$ \\
Conv2D & $3 \times 3$ & $109 \times 109 \times 64$ \\
MaxPooling2D & $2 \times 2$ & $54 \times 54 \times 64$ \\
Dropout & & $54 \times 54 \times 64$ \\
Conv2D & $3 \times 3$ & $52 \times 52 \times 128$ \\
AveragePooling2D & $2 \times 2$ & $26 \times 26 \times 128$ \\
Dropout & & $26 \times 26 \times 128$ \\
Conv2D & $1 \times 1$ & $26 \times 26 \times 128$ \\
Dropout & & $26 \times 26 \times 128$ \\
Flatten & & 86528 \\
Dense & & 256 \\
Dropout & & 256 \\
Dense & & 1 \\
\hline
\end{tabular}

割, 并在 Photoshop 中获取绿色环境比例作为标 签。将上述得到的训练样本和需要识别的街景图 片代人 CNN 模型中进行运算, 结果显示: 模型迭代 次数为 50 次时效果较好,训练集均方误差为 0.006 , 平均绝对误差为 0.053 ,测试集均方误差为 0.012 , 平 均绝对误差为 0.087 ,基本满足研究要求。

\section{2 实证分析}

\section{1 研究区域}

南京市是国内较早开始关注绿色视觉感知的 城市之一, 2002 年南京市启动“绿色南京”工程, 对 城市建成区内的立体绿化水平提出了明确目标 ${ }^{[62]}$ 。 本文选择南京市中心城区内 7 个区域、年代、类型等 条件差异明显的居住小区作为实证研究区域进行 调研(表 2)。调研时间为 2018 年 11-12 月,共发放 问卷 379 份, 其中有效问卷 359 份, 问卷有效率为 $94.72 \%$ 。有效问卷受访者中,女性样本数量略多于 男性; 平均年龄约 40 岁, 其中 19 59 岁受访者占总 数的 $78.49 \%$, 另有 $15.92 \%$ 的受访者为 60 岁以上的 老年人; $52.79 \%$ 以上受访者拥有本科以上学历; 家 庭年收人主要集中在 10 万元以下与 10 万 20 万元; 仅有 $15.64 \%$ 的受访者家庭拥有 1 辆以上的小汽车。

\section{2 研究框架与数据获取}

\subsection{1 实证研究框架}

基于绿色环境暴露对心理健康的影响框架提 
表 2 研究小区基础信息

Tab.2 Basic information of the study communities

\begin{tabular}{|c|c|c|c|c|c|c|}
\hline 小区名称 & 建设年代 & 房价/元 & 小区属性 & 距市中心距离/km & 楼层 & 有效问卷数 \\
\hline 五福里 & 1949年前 & 3 万以下 & 房改房/公房 & $0 \sim 5$ & 低层 & 35 \\
\hline 芳草园 & 1949-1998年 & 4 万以上 & 商品房 & $0 \sim 5$ & 高层 & 65 \\
\hline 居安里 & 1949-1998年 & 3 万 4 万 & 房改房/公房 & $0 \sim 5$ & 多层 & 63 \\
\hline 海棠园 & 1999年后 & 4 万以上 & 商品房 & $5 \sim 10$ & 多层 & 64 \\
\hline 莲花新城 & 1999年后 & 3 万以下 & 安置房/经适房 & $5 \sim 10$ & 高层 & 50 \\
\hline 云燕园 & 1949-1998年 & 3 万以下 & 房改房/公房 & $5 \sim 10$ & 多层 & 40 \\
\hline 银城东苑 & 1999年后 & 3 万 4 万 & 商品房 & $5 \sim 10$ & 高层 & 42 \\
\hline
\end{tabular}

出实证研究框架(图 3)。现有从单一个体视角出发 分析绿色环境对健康影响的研究已经比较成熟, 因 此, 本文主要从单一环境以及个体和环境交互的视 角出发构建分析模型, 研究不同绿色环境暴露测度 方法对心理健康的影响差异。其中,单一环境视角 选取了小区周边绿地率(模型 1)和小区周边绿视率 (模型 2)2 种测度指标, 个体和环境交互视角则选取 了居民绿色视觉暴露(模型 3 )进行测度,具体算法参 照 1.2 节。影响路径方面, 选取主观建成环境、社会 归属感、身体活动作为绿色环境暴露影响心理健康 的中介变量。主观建成环境会影响居民的身体活 动和社会归属感,对环境满意的居民容易有更高的 活动量, 并对所处环境产生更高的依赖和归属感。 归属感也可能影响居民的身体活动水平, 居民可能 更倾向于在归属感高的地方进行活动。此外,个人 及家庭属性也是影响心理健康的内在因素,因此作 为控制变量直接影响心理健康, 并同时纳人 3 个模 型的计算中。

2.2.2 问卷数据获取

受访者的心理健康状况采用中文版 The Short Warwick-Edinburgh Mental Wellbeing Scale(SWEMWBS)进行调查。该量表分别从“感到乐观/感觉有 价值/心情轻松/能处理好问题/思考问题清晰/和别 人关系亲近/能自己做决定” 共 7 个方面对受访者的
心理健康状况进行评估,每个问题均采用 1 分 $($ 从来 没有)到 5 分(一直都是)的形式进行调查。后期计算 中, 将所有题目得分相加并按照 Warwick Medical School 提供的分数转换表将原始项目得分转换为最 终评分。

中介变量中,受访者身体活动状况采用中文版 The International Physical Activity Questionnaire (IPAQ)-Short Form 进行调查,记录受访者最近一周 内的剧烈身体活动、适度身体活动和步行的时间。 后期计算中,按照相应规则对数据进行截断和重编 码, 计算受访者周身体活动总量, 并做归一化处 理。受访者归属感包括对社区的归属感和对南京 的归属感 2 个部分, 问题均采用 1 分(几乎没有)到 5 分(非常有)的形式进行调查。主观建成环境的调查 在已有文献基础上进行了一定补充,主要选取了美 观度、安全感、舒适度、安静感 4 个方面的问题[33-65], 问题均采用 1 分(非常不满意)到 5 分(非常满意)的 形式进行调查。

此外, 问卷还请受访者填写了居民的性别、年 龄、学历、家庭收人、是否有小汽车等个人及家庭属 性, 以及日常步行活动地点、活动路线及活动时间, 用于绿色视觉暴露的计算。

2.2.3 绿色环境暴露数据获取

在模型 1 和模型 2 中, 结合居民步行距离有关

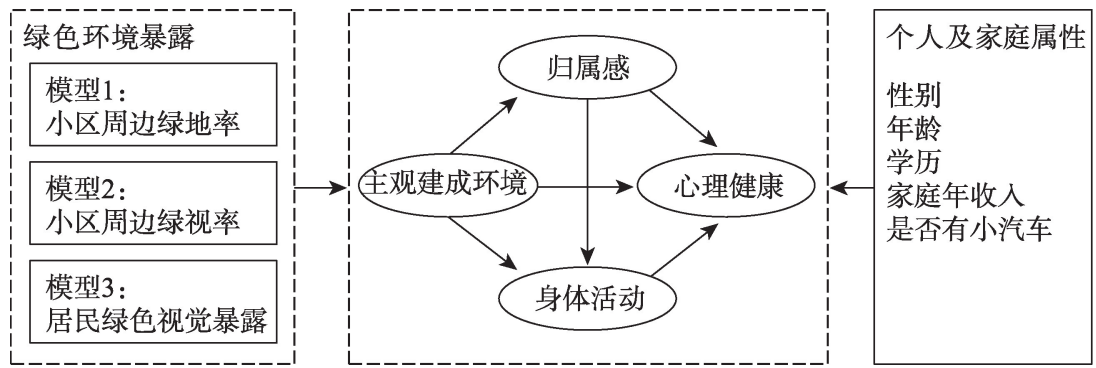

图 3 实证研究框架

Fig.3 Empirical research framework in Nanjing, China 
研究,选取小区边界向外 $950 \mathrm{~m}$ 缓冲区作为小区周 边绿地率和绿视率的绿色环境暴露分析范围 ${ }^{[6]}$ 。 其中, 绿地率用 NDVI值测算, 选取与街景地图同期 遥感数据,篮选 NDVI值大于 0.5 的区域作为绿地。 小区周边绿视率及街景图片来自腾讯静态街景图 片, 拍摄时间为 2014 年 12 月, 与问卷调研时间月份 基本符合。利用 Python 程序调用腾讯街景静态图 API 批量获取图片数据, 对小区周边道路选取均匀 的 $30 \mathrm{~m}$ 间断点作为观测点, 以平视视角调用图片。 研究共选择有效观测点 12325 个, 获取有效街景图 片 28723 张, 人工篮选后, 保留有效图片 26415 张。 由于平台返回不稳定, 存在部分图片缺失。各小区 周边绿地率和绿视率的值以及有效观测点和图片 的数量如表 3 所示, 小区的绿地率和绿视率之间没 有明显的相关性。

绿色视觉暴露按照 1.2 节中模型计算, 本文只 考虑居民日间步行出行活动, 计算中有效系数取 1 。以居安里小区为例, 图 4 显示了绿视率在小区周 边的分布和小区中一位居民的活动路线, 并在表 4 中列出了其 4 条活动路线的平均绿视率和出行时 长。经计算可得该居民的绿色视觉暴露值为 1055.04。为方便计算, 将所有居民的绿色视觉暴露
值统一做归一化处理。

\section{3 分析方法}

结构方程模型是一种兼有测量模型和分析模 型的常用分析手段,被广泛运用于社会、心理、教育 等多个领域, 是处理多因多果关系的重要工具 ${ }^{[67]}$ 。 本文基于实证研究框架,使用 $\mathrm{R}$ 语言建立结构方程 模型, 模型包含 16 个变量, 样本数量大于变量数量 的 20 倍, 满足模型分析要求。结构方程模型分为结 构模型和测量模型。对测量模型的信度及效度检 验结果显示, 所有变量 Cronbach's $\alpha$ 值均超过 0.6 的 可接受水平, 且测量模型的因子载荷均大于 0.6 ,证 明模型的信度和效度良好。表 5 对模型中除了个人 及家庭属性外的变量进行了说明。问卷中对体力 活动的调查包括剧烈活动时间、适度身体活动时间 和步行身体活动时间 3 个部分, 综合分析影响路径 显著性水平和模型整体拟合度后, 最终仅使用步行 身体活动时间作为测度。

\section{3 结果分析}

\section{1 描述性分析}

将居民个人及家庭属性和其余变量进行交叉

表 3 小区绿色环境暴露描述

Tab.3 Greenspace exposuredescription of the communities

\begin{tabular}{ccccc}
\hline 小区名称 & 小区周边绿地率/\% & 小区周边绿视率/\% & 有效观测点数量/个 & 有效图片数量/张 \\
\hline 五福里 & 15.07 & 24.00 & 2095 & 4282 \\
芳草园 & 6.60 & 28.14 & 1017 & 2092 \\
居安里 & 14.28 & 21.37 & 4305 & 10505 \\
海棠园 & 12.36 & 29.53 & 1879 & 4096 \\
莲花新城 & 15.51 & 16.50 & 1349 & 2128 \\
云燕园 & 22.17 & 21.36 & 825 & 1663 \\
银城东苑 & 10.26 & 35.81 & 855 & 1379 \\
\hline
\end{tabular}
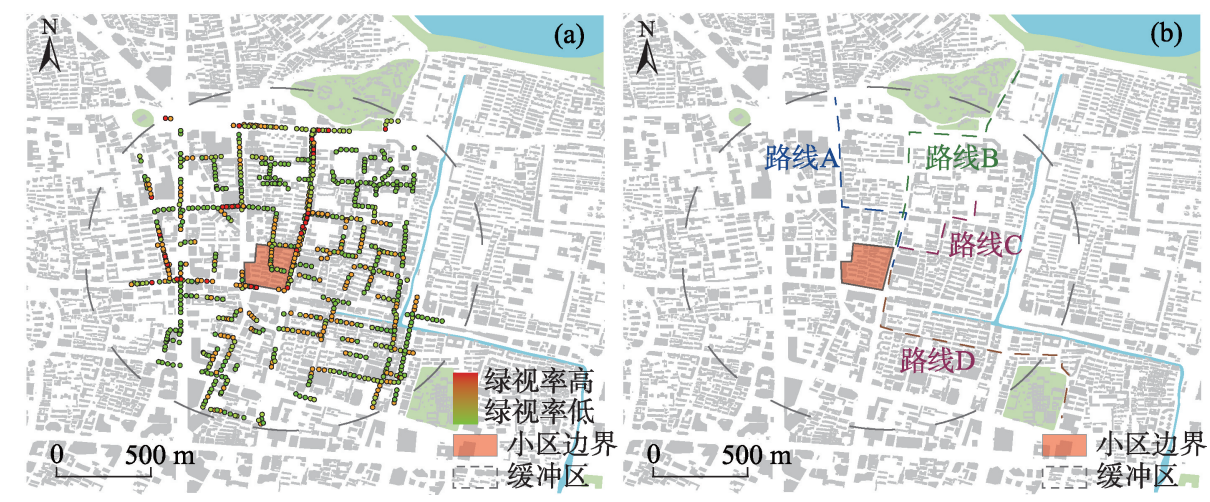

图 4 小区周边绿视率分布及居民活动路线示例

Fig.4 Example of green view index distribution and resident activity route around a community 
表4 居民绿色视觉暴露计算数据示例

Tab.4 Green visual exposure

\begin{tabular}{ccc}
\hline 居民活动路线 & 出行时长 $/ \mathrm{min}$ & 路线平均绿视率 $/ \%$ \\
\hline 路线 $\mathrm{A}$ & 10 & 24.12 \\
路线 $\mathrm{B}$ & 15 & 25.73 \\
路线 $\mathrm{C}$ & 6 & 23.15 \\
路线 $\mathrm{D}$ & 13 & 22.23 \\
\hline
\end{tabular}

制表分析(表6)。结果表明,女性心理健康水平略高 于男性; 随着年龄增长, 心理健康水平有上升趋势; 家庭年收人 50 万元以上人群心理健康程度明显高 于其他受访者;学历状况和是否拥有小汽车对心理 健康影响不大。绿色视觉暴露方面, 男性暴露量略 高于女性; 18 岁以下青少年由于日间步行时间普遍 偏短,绿色视觉暴露量普遍偏低; 家庭年收人 20 万 元以上的人群平均绿色视觉暴露量低于收人较低 人群;而不同学历居民的暴露量则差异不大。

不同年龄、学历、家庭收人水平的受访者身体 活动差异较大。1 18 岁以下的未成年人和 60 岁以上 的老年人身体活动水平均较高; 相较于拥有本科及 以上学历的受访者群体, 本科以下学历受访者有更 多的身体活动; 家庭收人在 20 万元以下受访者平均 身体活动量高于总体平均值, 更高收人家庭的群体 则低于平均值; 拥有小汽车的人群身体活动高于没 有小汽车的人群。归属感方面, 男性、学历和收人 较高的受访者对小区和南京的归属感普遍高于女 性、学历和收人较低的受访者; 60 岁及以上人群普 遍对南京的归属感更高,但是对社区的归属感较 差。受访者普遍对居住环境的美观度有更高的满 意度,对安全感和舒适度的体验则较差。老年人对 美观度和安全感感知更好, 但对舒适度和安静感感
知较差。本科以下学历受访者对环境各方面的感 知均优于本科及以上学历受访者。家庭年收人在 20 万 20 万元的受访者相对其它收人层次人群对安 全感评价更高;年收人 50 万元以上受访者则对美观 度、舒适度和安静感均更为满意。

\section{2 绿色环境暴露对心理健康的影响}

模型使用极大似然法进行估算,拟合指数验证 结果表明,选定的拟合指数中仅有比较拟合指数 (CFI)略低于参考值, 3 个模型的总体拟合程度良好 (表 7)。

表 8 给出了模型的标准化路径系数和显著性水 平。总体来看, 3 个模型中绿色环境暴露对心理健 康总效应和直接效应均呈现显著的正向影响,说明 较高的绿色环境暴露可以促进居民产生积极的心 理感受,与预期结果相符。但同时可以发现,在控 制了相同的个人及家庭属性的情况下, 3 个模型的 间接效应路径存在较大差异。

模型 1 和模型 2 的结果对比显示, 当测度指标 从二维的绿地率扩展到三维的绿视率时,绿色环境 暴露对心理健康的影响直接效应系数增大,且出现 了主观建成环境为中介变量的间接影响路径。间 接影响表现出了遮掩效应 ${ }^{[68]}$, 具体表现在尽管主观 建成环境对心理健康有显著正向影响,证实了良好 的环境感知可以促进居民产生积极的心理状态,但 小区周边绿视率对主观建成环境却表现出了显著 的负向影响。模型 3 中对绿色环境暴露的测度进一 步结合了居民日常活动因素, 此时绿色环境暴露对 心理健康的总效应和直接效应系数都显著增大。

结合图 5 可以看出,单一环境视角下,绿地率对 心理健康仅存在直接效应, 而对主观环境感知、身

表 5 模型部分变量描述

Tab.5 Partial model variable description

\begin{tabular}{|c|c|c|c|}
\hline 变量类别 & 潜变量 & 测量变量 & 测量变量类型及取值 \\
\hline \multirow[t]{3}{*}{ 解释变量 } & 绿色环境暴露 & 小区周边绿地率(模型 1) & 连续变量 \\
\hline & & 小区周边绿视率(模型 2) & 连续变量 \\
\hline & & 居民绿色视觉暴露(模型 3) & 连续变量 $(0 \sim 1)$ \\
\hline \multirow[t]{7}{*}{ 中介变量 } & 主观建成环境 & 我认为小区及周边绿化充足 & 有序分类变量(1 5) \\
\hline & & 我认为在小区及周边步行是安全的 & \\
\hline & & 我认为在小区及周边步行是舒适的 & \\
\hline & & 我认为小区及周边环境安静 & \\
\hline & 身体活动 & IPAQ 中文版短表周身体活动总量 & 连续变量 $(0 \sim 1)$ \\
\hline & 归属感 & 我对现在居住的小区有归属感 & 有序分类变量(1 5) \\
\hline & & 我对南京有归属感 & \\
\hline 因变量 & 心理健康 & SWEMWBS 中文版心理健康评分 & 有序分类变量(7 35) \\
\hline
\end{tabular}


表 6 居民个人及家庭属性与模型其余变量的交叉分析

Tab.6 Cross-analysis of residents' personal and family attributes and other variables of the model

\begin{tabular}{|c|c|c|c|c|c|c|c|c|c|}
\hline \multirow{2}{*}{$\begin{array}{l}\text { 变量 } \\
\text { 名称 }\end{array}$} & \multirow{2}{*}{$\begin{array}{l}\text { 心理 } \\
\text { 健康 }\end{array}$} & \multirow{2}{*}{$\begin{array}{c}\text { 绿色视觉 } \\
\text { 暴露 }\end{array}$} & \multirow{2}{*}{$\begin{array}{c}\text { 身体活动 } \\
\text { 总量 }\end{array}$} & \multicolumn{2}{|c|}{ 归属感 } & \multicolumn{4}{|c|}{ 主观建成环境 } \\
\hline & & & & 社区 & 南京 & 美观度 & 安全感 & 舒适度 & 安静感 \\
\hline \multicolumn{10}{|l|}{ 性别 } \\
\hline 男 & $24.24(4.93)$ & $0.47(0.15)$ & $0.28(0.24)$ & $2.57(0.88)$ & $3.02(0.93)$ & $2.80(1.17)$ & $2.56(1.07)$ & $2.35(0.97)$ & $2.70(1.13)$ \\
\hline 女 & $24.64(4.98)$ & $0.41(0.13)$ & $0.29(0.26)$ & $2.35(0.94)$ & $2.92(1.06)$ & $2.57(1.08)$ & $2.37(1.07)$ & $2.53(0.96)$ & $2.53(1.13)$ \\
\hline \multicolumn{10}{|l|}{ 年龄 } \\
\hline 18 岁及以下 & $23.32(5.59)$ & $0.34(0.18)$ & $0.33(0.26)$ & $2.35(1.11)$ & $2.70(1.01)$ & $2.30(1.20)$ & $2.35(1.20)$ & $2.50(1.16)$ & $2.75(1.22)$ \\
\hline 19 59岁 & $24.34(4.84)$ & $0.45(0.20)$ & $0.26(0.23)$ & $2.51(0.88)$ & $2.98(0.96)$ & $2.70(1.08)$ & $2.46(1.03)$ & $2.48(0.94)$ & $2.70(1.60)$ \\
\hline 60 岁及以上 & $25.29(5.10)$ & $0.44(0.20)$ & $0.39(0.30)$ & $2.23(0.94)$ & $3.04(1.12)$ & $2.77(1.30)$ & $2.53(2.24)$ & $2.23(1.03)$ & $2.18(1.11)$ \\
\hline \multicolumn{10}{|l|}{ 学历 } \\
\hline 本科以下 & $24.65(4.97)$ & $0.44(0.19)$ & $0.35(0.28)$ & $2.36(0.96)$ & $2.91(1.07)$ & $2.78(1.19)$ & $2.50(1.14)$ & $2.46(1.03)$ & $2.62(1.17)$ \\
\hline 本科及以上 & $24.25(4.92)$ & $0.43(0.15)$ & $0.23(0.20)$ & $2.55(0.86)$ & $3.02(0.91)$ & $2.61(1.07)$ & $2.43(1.01)$ & $2.43(0.92)$ & $2.61(1.09)$ \\
\hline \multicolumn{10}{|l|}{ 家庭年收人 } \\
\hline 10 万元以下 & $24.19(4.63)$ & $0.45(0.18)$ & $0.30(0.27)$ & $2.50(0.92)$ & $2.97(1.04)$ & $2.69(1.15)$ & $2.46(1.09)$ & $2.43(0.93)$ & $2.47(1.11)$ \\
\hline 10 万 20 万元 & $24.84(4.94)$ & $0.47(0.17)$ & $0.31(0.25)$ & $2.36(0.85)$ & $2.92(0.95)$ & $2.66(1.11)$ & $2.44(1.06)$ & $2.48(1.04)$ & $2.77(1.21)$ \\
\hline 20 万 50 万元 & $23.68(5.46)$ & $0.37(0.14)$ & $0.24(0.20)$ & $2.43(0.93)$ & $2.95(0.94)$ & $2.60(1.10)$ & $2.52(1.10)$ & $2.37(0.97)$ & $2.60(1.00)$ \\
\hline 50 万元以上 & $27.11(4.70)$ & $0.39(0.13)$ & $0.23(0.21)$ & $2.94(1.09)$ & $3.41(1.00)$ & $3.24(1.20)$ & $2.47(1.07)$ & $2.59(0.87)$ & $2.88(1.11)$ \\
\hline \multicolumn{10}{|l|}{ 是否拥有汽车 } \\
\hline 有小汽车 & $24.70(4.81)$ & $0.44(0.20)$ & $0.32(0.27)$ & $2.51(0.90)$ & $2.99(1.00)$ & $2.60(1.08)$ & $2.47(1.05)$ & $2.43(0.94)$ & $2.70(1.13)$ \\
\hline 无小汽车 & $24.03(5.15)$ & $0.43(0.19)$ & $0.26(0.24)$ & $2.38(0.92)$ & $2.94(0.99)$ & $2.82(1.20)$ & $2.45(1.12)$ & $2.47(1.02)$ & $2.49(1.12)$ \\
\hline 总体 & $24.44(4.95)$ & $0.44(0.20)$ & $0.29(0.25)$ & $2.46(0.91)$ & $2.97(0.99)$ & $2.69(1.13)$ & $2.46(1.07)$ & $2.44(0.97)$ & $2.62(1.13)$ \\
\hline
\end{tabular}

注:表中数值为平均值(标准差)。

表 7 模型拟合指数

Tab.7 Goodness-of-fit statistics of the model

\begin{tabular}{ccccc}
\hline 模型 & $\chi^{2} / \mathrm{DF}$ & GFI & CFI & RMESA \\
\hline 模型 1 & 2.610 & 0.908 & 0.842 & 0.067 \\
模型 2 & 2.768 & 0.905 & 0.832 & 0.070 \\
模型 3 & 2.596 & 0.910 & 0.849 & 0.067 \\
参考值 & $1 \sim 3$ & $>0.90$ & $>0.90$ & $<0.08$ \\
\hline
\end{tabular}

表 8 绿色环境暴露对心理健康影响效应

Tab.8 Effects of greenspace exposure on mental health

\begin{tabular}{cccc}
\hline 影响 & 模型 1 & 模型 2 & 模型 3 \\
\hline 总效应 & $0.168^{* *}$ & $0.124^{* *}$ & $0.286^{*}$ \\
直接效应 & $0.168^{* *}$ & $0.185^{* *}$ & $0.237^{* * *}$ \\
间接总效应 & - & $-0.061^{* * *}$ & $0.049^{*}$ \\
\hline
\end{tabular}

注: ***、***分别表示 $P<0.05 、 P<0.01 、 P<0.001$ 。

体活动和归属感的间接路径均不显著。相对于绿 地率, 绿视率将绿色环境测度扩展到了三维感知层 面, 从个体与环境交互视角更加真实地反映出了居 民对绿色环境的感知状况, 在模型中的主观建成环 境路径表现出显著影响。更进一步,主观建成环境 和身体活动都可以作为绿色视觉暴露影响心理健 康的中介变量。居民日常活动中实际可能接收到
的绿色信息总量越高,对周边环境的感知可能越 好,进而积极心理健康水平也越高, 因此绿色视觉 暴露可以通过影响主观建成环境影响居民心理健 康; 同样, 居民日常活动中长时间暴露在高绿视率 的环境下可能更容易有更多的身体活动,进而可以 促进心理健康水平的提升。

\section{4 结论与讨论}

本文首先基于现有研究提出了绿色环境暴露 对心理健康影响研究的理论框架,综合考虑了不同 视角下的绿色环境暴露指标对心理健康的影响路 径差异。在绿色环境暴露的测度方面,在现有研究 单一个体或者单一环境的视角基础上进行扩展, 关 注了个体与环境交互关系,将传统的二维绿色环境 测度扩展到了三维层面,利用静态街景数据结合机 器学习的方法弥补了对视觉感知关注的不足,并进 一步将人群实际活动纳人考量。在影响路径方面, 关注了不同的绿色环境暴露指标对心理健康影响 路径存在差异的可能性,对现有研究有一定补充。

其次,本文以南京市为实证对象,选取了小区 
(a) 模型1结果路径

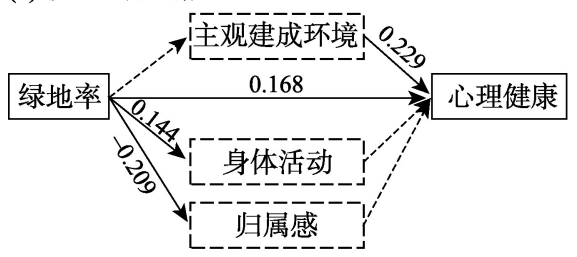

(b) 模型2结果路径

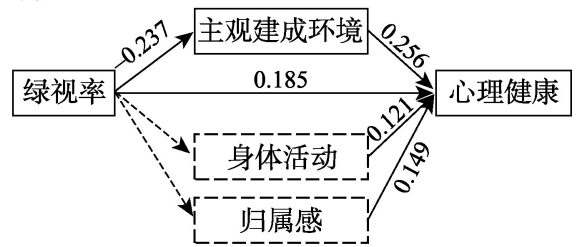

(c) 模型 3 结果路径

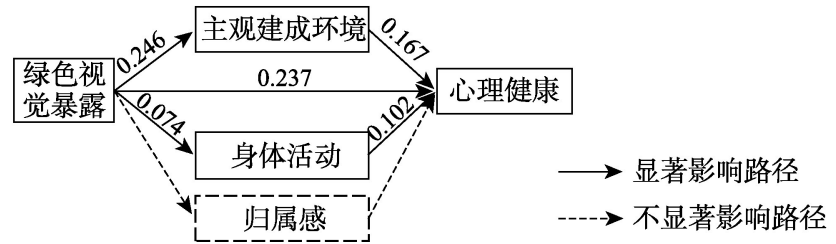

图 53 个模型影响路径

Fig.5 Impact path diagram of the three models

周边绿地率、小区周边绿视率、居民绿色环境暴露 总量 3 个指标分别建立结构方程模型, 分析绿色环 境暴露对心理健康的影响。主要结论有: (1) 从单一 环境视角及个体和环境交互视角选取的 3 种绿色环 境暴露测度指标对居民心理健康均有显著正向影 响, 控制其余变量相同情况下, 绿色视觉暴露对心 理健康的直接影响系数最大, 绿视率次之, 绿地率 则最小。(2) 不同绿色环境暴露测度指标对心理健 康的影响路径存在差异。其中, 主观建成环境可以 作为绿视率和绿色视觉暴露对心理健康影响的中 介变量, 身体活动仅是绿色视觉暴露对心理健康影 响的中介变量。这一结果更加清晰地反映了环境 对心理健康的影响机制, 侧面说明了多角度选取绿 色环境测度指标的重要性。

上述方法和结论对城市的绿地规划建设管理 具有一定参考价值。在绿地规划中, 仅仅从单一视 角考虑是有局限的, 应结合城市自身情况, 综合考 虑宏观与微观、二维与三维、静态与动态等不同类 型的指标, 建立对城市绿地的综合管理体系。在建 设和评估过程中应充分考虑人的视角, 从居民实际 需求角度完善。在以人为本的背景下, 未来从个体 与环境交互的视角结合交通学、社会学、医学等多 个领域提出绿地系统规划方案和制定相关政策将 会愈发普遍, 例如对人群移动性、环境敏感度等因 素的综合分析, 以及对特殊人群的关注等。

本文还存在不足之处。(1) 在绿色视觉暴露的 分析中,本文的基本假设是 “居民会持续地从周围 环境中接收(绿色)信息”,但居民接收信息的强度可 能受到居民主观意愿和活动的影响, 例如居民在锻 炼时对周边环境的敏感程度可能与通勤途中有很
大差别。(2)对环境暴露的测算中,本文只关注了街 道绿色环境的视觉暴露,缺少对住宅和停留地点内 部绿色环境的测算。(3)计算方法方面,本文在绿色 视觉暴露计算公式中提出了有效系数的概念, 用于 测度不同时空行为状态下对环境感知强度的差异, 但在实际操作中没有提出系统合理的计算方法。 上述问题主要受限于更为精细的数据获取能力, 一 些新的数据获取方式(如VR 实验、传感器测算)可能 对后续研究进一步完善提供帮助。

致谢: 对在本文技术上给予大力支持的南京大学建 筑与城市规划学院祁毅老师以及参与问卷调查的 各位同学表示由衰的感谢。

\section{参考文献(References)}

[1] 李小平.一个新的交叉学科:环境暴露学 [J]. 国外医学地 理分册, 2016, 37(2): 81-84. [Li Xiaoping. Environmental exposure science: A new inter discipline. Foreign Medical Science Section of Medgeography, 2016, 37(2): 81-84. ]

[2] Nieuwenhuijsen M J. Urban and transport planning, environmentalexposures and health- new concepts, methodsand tools to improve health in cities [J]. Environmental Health, 2014, 15: 161-171.

[3] 马静, 柴彦威, 符婷婷. 居民时空行为与环境污染暴露对 健康影响的研究进展 [J]. 地理科学进展, 2017, 36(10): 1260-1269. [Ma Jing, Chai Yanwei, Fu Tingting. Progress of research on the health impact of people's space-time behavior and environmental pollution exposure. Progress in Geography, 2017, 36(10): 1260-1269. ]

[4] 张莉, 王五一, 廖永丰. 城市空气质量健康风险评估研究 进展 [J]. 地理科学进展, 2006, 25(3): 39-47. [Zhang Li, Wang Wuyi, Liao Yongfeng. Research progress on urban 
air quality health risk assessment. Progress in Geography, 2006, 25(3): 39-47. ]

[5] Sugiyama T, Carver A, Koohsari M J, et al. Advantages of public green spaces in enhancing population health $[\mathrm{J}]$. Landscape and Urban Planning, 2018, 178: 12-17.

[6] 王兰, 廖舒文, 王敏. 影响呼吸系统健康的城市绿地空间 要素研究: 以上海市某中心区为例 [J]. 城市建筑, 2018, 24(3): 10-14. [Wang Lan, Liao Shuwen, Wang Min. The impact of spatial factors of urban green apace on respiratory health: A case of a central district in Shanghai. Urbanism and Architecture, 2018, 24(3): 10-14. ]

[7] Boers S, Hagoort K, Scheepers L, et al. Does residential green and blue space promoterecovery in psychotic disorders? A cross- sectionalstudy in the province of Utrecht, The Netherlands [J]. International Journal of Environmental Research and Public Health, 2018, 15: 1-8.

[8] Schwartz R M, Christina N, Gillezeau, et al. Longitudinalimpact of hurricane sandy exposure onmental health symptoms [J]. International Journal of Environmental Research and Public Health, 2017, 14: 1-12.

[9] Dzhambov A M, Markevych I, Hartig T, et al. Multiple pathways link urban green- and bluespace to mental health inyoung adults [J]. Environmental Research, 2018, 166: 223-233.

[10] Wang W, Lin Z Y, Zhang L W, et al. Building visual green index: A measure of visual green spaces for urbanbuilding [J]. Urban Forestry \& Urban Greening, 2019, 40: 335-343.

[11] Huang J, Deng F, Wu S W, et al. Comparisons of personal exposure to $\mathrm{PM}_{2.5}$ and $\mathrm{CO}$ by different commuting modes in Beijing, China [J]. Science of the Total Environment, 2012, 425: 52-59.

[12] 陈曦, 冯建喜. 基于步行性与污染物暴露空间格局比较 的建成环境健康效应: 以南京为例 $[\mathrm{J}]$. 地理科学进展, 2019, 38(2): 296-304. [Chen Xi, Feng Jianxi. Health effects of built environment based on a comparison of walkability and air pollution: A case study of Nanjing City. Progress in Geography, 2019, 38(2): 296-304. ]

[13] Semenza J C, M. P. H, Bubin C H, et al. Heat-related deaths during the July 1995 heat wave in Chicago [J]. The New England Journal of Medicine, 1996, 335(2): 84-90.

[14] Hajat S, Kovats R S, Lachowycz K. Heat-related and cold- related deaths in England and Wales: Who is at risk? [J]. Occupational and Environmental Medicine, 2007, 64: 93-100.

[15] 谢盼, 王仰麟, 彭建, 等. 基于居民健康的城市高温热浪 灾害脆弱性评价: 研究进展与框架 [J]. 地理科学进展, 2015, 34(2): 165-174. [Xie Pan, Wang Yanglin, Peng Ji- an, et al. Health related urban heat wave vulnerability assessment: Research progress and framework. Progress in Geography, 2015, 34(2): 165-174. ]

[16] Bowler D E, Buyung-Ali L M, Knight T M, et al. A systematic review of evidence for the added benefits to health of exposure to natural environments $[\mathrm{J}]$. BMC Public Health, 2010, 456: 1-10.

[17] Coon J T, Boddy K, Stein K, et al. Does participating in physical activity in outdoor natural environments have a greater effect on physical and mental wellbeing than physical activity indoors? A systematic review [J]. Community Health, 2011, 65: A1-A40.

[18] Capaldi C A, Dopko R L, Zelenski J M. The relationship between nature connectedness andhappiness: A metaanalysis [J]. Frontiers in Psychology, 2014, 5: 1-15.

[19] Bodin M, Hartig T. Does the outdoor environment matter for psychologicalrestoration gained through running? [J] Psychology of Sport and Exercise, 2003, 4: 141-153.

[20] Kerr J H, Fujiyama H, Sugano A, et al. Psychological responses to exercising in laboratoryand natural environments [J]. Psychology of Sport and Exercise, 2006, 7: 345-359.

[21] Johnson B S, Malecki K M, et al. Exposure to neighborhood green space and sleep: Evidence from the survey of the health of Wisconsin [J]. Sleep Health, 2018, 4: 413419.

[22] Gidlow C, Smith G, Kempen E V, et al. Development of the Natural Environment Scoring Tool (NEST) [J]. Urban Forestry \& Urban Greening, 2017, 29: 322-333.

[23] 谭冰清, 武书帆, 苏世亮, 等. 城市公共绿地供给与居民 健康的空间关联 [J]. 城市建筑, 2018, 24(8): 57-61. [Tan Bingqing, Wu Shufan, Su Shiliang, et al. Spatial association of urban public green space supply and residents' health. Urbanism and Architecture, 2018, 24(8): 57-61.

[24] 姚崇怀, 李德玺. 绿容积率及其确定机制 [J]. 中国园 林, 2015, 31(9): 5-11. [Yao Chonghuai, Li Dexi. Study on the Green Volume Ratio (GVR) and its calculating mechanism. Chinese Landscape Architecture, 2015, 31 (9): 5-11. ]

[25] 肖希, 韦怡凯, 李敏. 日本城市绿视率计量方法与评价 应用 [J]. 国际城市规划, 2018, 33(2): 98-103. [Xiao Xi, Wei Yikai, Li Min. The method of measurement and applications of visible green index in Japan. Urban Planning International, 2018, 33(2): 98-103. ]

[26] 青木陽二. 視野の広がりと緑量感の [J]. 造園雑誌, 1987, 51(1): 1-10. [Yoji Aoki. Relationship between percieved greenery and width of visual fields. The Japanese 
Institute of Landscape Architecture, 1987, 51(1): 1-10. ]

[27] 古澤, 加藤. 真夏日の不快感を緩和する都市の緑の 景観・心理効果について〜都市の緑量と心理的効果 の相関関係の社会実験調査について $[\mathrm{EB} / \mathrm{OL}]$. 国土 交通省. 2005-08-12 [2020-02-13]. http://www.mlit.go.jp/ kisha/kisha05/04/040812_3/01.pdf. [Furusawa, Kato. About the green landscape and psychological effects of the city to alleviate the discomfort of midsummer sun: Social experiment research on the correlation between urban greenness and psychological effects. Ministry of Land, Infrastructure, Transport and Tourism, 2005-08-12 [2020-02-13]. http://www.mlit.go.jp/kisha/kisha05/04/04 0812 3/01.pdf. ]

[28] 矢川麻紀子, 田村明弘. 感覚時間による場と人との交 感作用の指標化 [J]. 日本建築学会枝術報告集, 1999 (8): 155- 158. [Makiko Vagawa, Akihiro Tamura. Human's sense of time: As an index of the sympathy between man and environment. Architectural Institute of Japan, 1999(8): 155-158. ]

[29] 藤井健史, 山田悟史. 廣瀬德郎, 等. CG モデルによる 全方位緑視率の計量手法とその応用可能性 [R].日本 建築学会枝術報告集，2013，19(43)：1067-1072. [Takeshi Fujii, Satoshi Yamada, Tokuro Hirose, et al. Measurement of the ratio of visible green spaces in the omnidirectional field of vision using CG models and their potential applications. Architectural Institute of Japan, 2013, 19(43): 1067-1072. ]

[30] 赵庆, 唐洪辉, 魏丹, 等. 基于绿视率的城市绿道空间绿 量可视性特征 [J]. 浙江农林大学学报, 2016, 33(2): 288-294. [Zhao Qing, Tang Honghui, Wei Dan, et al. Spatial visibility of green areas of urban greenway using the green appearance percentage. Journal of Zhejiang Agriculture and Forestry University, 2016, 33(2): 288-294. ]

[31] 李凤霞, 石辉, 撒利伟, 等. 微分法三维激光点云数据单 木三维绿量测定研究 [J]. 西安建筑科技大学学报(自然 科学版), 2017, 49(4): 530-535. [Li Fengxia, Shi Hui, Sa Liwei, et al. 3D green volume measurement of single tree using 3D laser point cloud data and differential method. Journal of Xi'an University of Architecture \& Technology, 2017, 49(4): 530-535. ]

[32] 崔喆, 何明怡, 陆明. 基于街景图像解译的寒地城市绿 视率分析研究: 以哈尔滨为例 [J]. 中国城市林业, 2018, 16(5): 38-42. [Cui Zhe, He Mingyi, Lu Ming. An analysis of green view index in cold region city: A case study of Harbin. Journal of Chinese Urban Forestry, 2018, 16(5): 38-42. ]

[33] 徐碟青, 孟若希, 黄舒晴, 等. 疗愈导向的街道设计: 基 于 VR 实验的探索 [J]. 国际城市规划, 2019, 34(1): 38-
45. [Xu Leiqing, Meng Ruoxi, Huang Shuqing, et al. Healing oriented street design: An exploration based on VR experiments. Urban Planning International, 2019, 34 (1): 38-45. ]

[34] Kwan M. From place- based to people- based exposure measures [J]. Social Science \& Medicine, 2009, 69: $1311-1313$

[35] Grahn P, Stigsdotter U A. Grahn P, et al. Landscape planning and stress $[\mathrm{J}]$. Urban Forestry \& Urban Greening, 2003, 2(1): 1-18.

[36] Nielsen T S, Hansen K B. Do green areas affect health? Results from a Danish survey onthe use of green areas and health indicators $[\mathrm{J}]$. Health \& Place, 2006, 13: 839850 .

[37] Berg M V D, Poppel M V P, Smith G, et al. Does time spent on visits to green space mediate the associations betweenthe level of residential greenness and mental health? [J]. Urban Forestry \& Urban Greening, 2017, 25: 94-102.

[38] Zhang L, Zhou S H, Kwan M, et al. Impacts of individual daily greenspace exposure on health based on individual activity space and structural equation modeling [J]. International Journal of Environmental Research and Public Health, 2018, 15: 1-18.

[39] Gascon M, Triguero-Mas M, Martínez D, et al. mental health benefits of long-term exposure to residential green and blue spaces: A systematic review [J]. International Journal of Environmental Research and Public Health, 2015, 12(4): 4354-4379.

[40] Paluska S A, Schwenk T L. Physical activity and mental health [J]. Sports Medicine, 2000, 29(3): 167-180.

[41] Evans W P, Owens P, Marsh S C, et al. Environmental factors, locus of control, and adolescent suicide risk [J]. Child and Adolescent Social Work Journal, 2005, 22(3): 301-319.

[42] Rappe E, Kivelä S, Rita H. Visiting outdoor green environments positively impacts self-rated health among older people in long-term care [J]. Hort Technology, 2006, 16(1): 55-59.

[43] Akpinar A, Barbosa-Leiker C, Brooks K A. Does green space matter? Exploring relationships between greenspacetype and health indicators [J]. Urban Forestry \& Urban Greening, 2016, 20: 407-418.

[44] Helbich M, Klein N, Roberts H, et al. More green space is related to less antidepressant prescription rates in the Netherlands: A Bayesian geoadditive quantile regression approach [J]. Environmental Research, 2018, 166: 290297. 
[45] Liu Q Y, Zhang Y J, Lin Y W, et al. The relationship between self- rated naturalness of university green space and students' restoration and health $[\mathrm{J}]$. Urban Forestry \& Urban Greening, 2018, 34:259-268.

[46] Thompson C W, Roe J, Aspinall P, et al. More green space is linked to less stress in deprived communities:Evidence from salivary cortisol patterns $[\mathrm{J}]$. Landscape and Urban Planning, 2012, 105: 221-229.

[47] Yuan L, Shin K, Managi S. Subjective well-being and environmental quality: The impact of air pollution and green coverage in China $[\mathrm{J}]$. Ecological Economics, 2018, 153: 124-138.

[48] Pietilä M, Neuvonen M, Borodulin K, et al. Relationships between exposure to urban green spaces, physicalactivity and self-rated health [J]. Journal of Outdoor Recreation and Tourism, 2015, 10: 44-54.

[49] Lachowycz K, Jones A P. Towards a better understanding of the relationship between greenspaceand health: Development of a theoretical framework [J]. Landscape and Urban Planning, 2013, 118: 62-69.

[50] Reid C E, Kubzansky L D, Li J, et al. It's not easy assessing greenness: A comparison of NDVI datasets and neighborhood types and their associations with self-rated health in New York City [J]. Health \& Place, 2018, 54: 92-101.

[51] Dadvand P, Bartoll X, Basagaña X, et al. Green spaces and general health: Roles of mental health status, socialsupport, and physical activity [J]. Environment International, 2016, 91: 161-167.

[52] Lee S, Ellis C D, Kweon B, et al. Relationship between landscape structure and neighborhoodsatisfaction in urbanized areas [J]. Landscape and Urban Planning, 2008, 85: $60-70$

[53] $\mathrm{Lu} \mathrm{Y}$. Using google street view to investigate the association between streetgreenery and physical activity $[\mathrm{J}]$. Landscape and Urban Planning, 2018, 8: 2-9.

[54] Rzotkiewicz A, Pearson A L, Dougherty B V, et al. Systematic review of the use of google street view in health research: Major themes, strengths, weaknesses and possibilities for future research [J]. Health \& Place, 2018, 52: 240-246.

[55] 小宫佑登, 須㟝純一. 航空機計測を用いた市街地にお ける緑視率推定 [J]. 土木学会論文集 D1, 2015, 71(1): 1-9. [Yuto KOMIYA, Junichi SUSAKI. Estimation of visible green index in urban area using airborne measurement. Journal of Japan Society of Civil Engineers, Ser. D1, 2015, 71(1): 1-9. ]

[56] Li X J, Ratti C, Seiferling I. Mapping urban landscapes along streets using google street view [C]// Peterson M P. Advances in cartography and GIScience: Selections from the international cartographic conference 2017. Washington D C, USA: Springer, Cham, 2017: 341-356.

[57] Helbich M, Yao Y, Liu Y, et al. Using deep learning to examine street view green and blue spaces and their associations with geriatric depression in Beijing, China [J]. Environment International, 2019, 126: 107-117.

[58] 彭锐, 刘海霞. 城市道路绿视率自动化计算方法研究 [J]. 北京规划建设, 2018(4): 61-64. [Peng Rui, Liu Haixia. Research on automatic calculation method of green line rate of urban roads. Beijing Planning Review, 2018 (4): 61-64. ]

[59] 卢官明, 何嘉利, 间静杰, 等.一种用于人脸表情识别的 卷积神经网络 [J]. 南京邮电大学学报, 2016, 36(1): 1622. [Lu Guanming, He Jiali. Yan Jingjie, et al. Convolutional neural network for facial expression recognition. Journal of Nanjing University of Posts and Telecommunications, 2016, 36(1): 16-22. ]

[60] 崔璐, 张鹏, 车进. 基于深度神经网络的遥感图像分类 算法综述 [J]. 计算机科学, 2018, 45(6A): 50-53. [Cui Lu, Zhang Peng, CheJin. Overview of deep neural network based classification algorithms for remote sensing images. Computer Science, 2018, 45(6A): 50-53. ]

[61] 何宛余, 李春, 聂广洋, 等. 深度学习在城市感知的应用 可能: 基于卷积神经网络的图像判别分析 [J]. 国际城 市规划, 2019, 34(1): 8-17. [He Wanyu, Li Chun, NieGuangyang, et al. The latent application of deep learning in urban perception: Image discrimination analysis by convolutional neural network. Urban Planning International, 2019, 34(1): 8-17. ]

[62] 解自来. 提高主城 “绿视率” 是建设 “绿色南京”的重要 环节 [J]. 现代城市研究, 2003(6): 83-86. [Xie Zilai. Raising the proportion of greenness of main city is an important tacheto build greening Nanjing. Modern Urban Research, 2003(6): 83-86. ]

[63] Arango C M, Paez D C, Reis R S, et al. Association between the perceived environment and physical activity among adults in Latin America: A systematic review [J]. International Journal of Behavioral Nutrition and Physical Activity, 2013, 10: 122. doi: 10.1186/1479-5868-10-122.

[64] 冯建喜, 黄旭, 汤爽爽. 客观与主观建成环境对老年人不 同体力活动影响机制研究: 以南京为例 [J]. 上海城市 规划, 2017(3): 17-23. [Feng Jianxi, Huang Xu, Tang Shuangshuang. Comparing the influences of objective and subjective built environments on physical activities participation among the elderly: A case study of Nanjing, China. Shanghai Urban Planning Review, 2017(3): 17-23. ] 
[65] 姜玉培, 甄峰, 王文文, 等. 城市建成环境对居民身体活 动的影响研究进展与启示 [J]. 地理科学进展, 2019, 38 (3): 357-369. [Jiang Yupei, Zhen Feng, Wang Wenwen, et al. Influence of urban built environment on residents' physical activity: Review and implications. Progress in Geography, 2019, 38(3): 357-369. ]

[66] 王宁, 杜豫川. 社区居民适宜步行距离阈值研究 [J]. 交 通运输研究, 2015(2): 20-24, 30. [Wang Ning, Du Yuchuan. Resident walking distance threshold of community. Transport Research, 2015(2): 20-24, 30. ]
[67] 温忠麟, 张雷, 侯杰泰, 等. 中介效应检验程序及其应用 [J]. 心理学报, 2004, 36(5): 614-620. [Weng Zhonglin, Zhang Lei, Hou Jietai, et al. Testing and application of the mediating effects. Acta Psychologica Sinica, 2004, 36 (5): 614-620. ]

[68] 温忠麟, 叶宝娟. 中介效应分析: 方法和模型发展 [J]. 心理科学进展, 2014, 22(5): 731-745. [Wen Zhonglin, Ye Baojuan. Analyses of mediating effects: The development of methods and models. Advances in Psychological Science, 2014, 22(5): 731-745. ]

\title{
Impact of greenspace exposure on residents' mental health: A case study of Nanjing City
}

\author{
LI Zhixuan, HE Zhongyu*, ZHANG Yiming, JIN Shuangshuang, WANG Xuemei, \\ ZHU Jie, LIU Shicen \\ (School of Architecture and Urban Planning, Nanjing University, Nanjing 210093, China)
}

\begin{abstract}
The impact of greenspace exposure on mental health has long been the focus of scholars in different fields in China and internationally. Most studies are based on a single individual perspective, such as activity time, range of activities, and so on, or a single environmental perspective, such as the number of green spaces, green space accessibility, among others. Few studies have measured individuals' perception of the environment from the perspective of the interaction between the individuals and the environment. This study constructed a conceptual framework for the impact of green environment exposure on mental health based on considerations of residents' visual perception and spatiotemporal activities. In addition, this study also proposed a green visual exposure measurement method based on green view index and individual spatiotemporal activity, and estimated the green rate using convolutional neural network model and machine learning. This study took Nanjing City as an empirical research object, and used structural equation modeling to compare and analyze the differences in the impact of green rate, green view index, and total green visual exposure on mental health. In addition to observing the direct influences, environmental perception, physical activity, and sense of belongingness were selected as mediating variables to analyze the pathways of different indicators that affect mental health. The results show that the three greenspace exposure measurement indicators have a significant correlation with mental health, but the degree of influence and pathway are different. It is important to establish a more comprehensive green environment exposure evaluation index system. Subjective built- up environment perception can be used as a mediator of the impact of green view index and total green visual exposure on mental health. Physical activity only serves as a mediator of the impact of the total amount of green visual exposureon mental health. This study expands the research framework of the impact of greenspace exposure on mental health, and has important reference value for the planning and management of urban greenspace system.
\end{abstract}

Keywords: environmental exposure; green visual exposure; green view index; mental health; machine learning; Nanjing City 